\title{
Learning and Transition of Symbols: Towards a Dynamical Model of a Symbolic Individual
}

\author{
Takashi Hashimoto and Akira Masumi \\ School of Knowledge Science \\ Japan Advanced Institute of Science and Technology (JAIST) \\ 1-1, Nomi, Ishikawa, Japan, 923-1292 \\ hash@jaist.ac.jp, a-masumi@jaist.ac.jp
}

\begin{abstract}
We aim at constructing a model for an agent showing symbolic activities. Based on a discussion of the concept of a symbolic system we propose correspondences between symbolic activities and the behaviour of dynamical systems in which attractors develop in response to inputs. We examine correspondences such as, symbols to attractors, symbol manipulations to transitions among attractors, and regularities in symbol manipulation to the order of the transitions. By using a dynamical system showing transitions among attractors, we may be able to represent symbolic activities, in part. We try to construct such a system with a kind of chaotic neural networks, derived from the Hopfield model. We confirm that this system has multi-attractors and shows transitions among attractors in some parameter regions. Further, we investigate the response of the system to certain external inputs. Examining these simulation results suggests that this system learnt in a certain environment can be used as a model of a symbolic agent for the study of the emergence of linguistic communication.
\end{abstract}

\section{Introduction}

The remarkable feature of linguistic communications is the use of symbols for transmitting information and mutual understanding. Deacon [4] pointed out that humans are symbolic species, namely, we show symbolic cognitive activities such as learning, formation, and manipulation of symbols. In research into the origin and the evolution of language, we should elucidate the emerging process of such symbolic cognitive activities.

Most agent models in simulation studies of language evolution presuppose symbol processing ability [3]. For example, a computational model for the evolution of compositional syntax introduced in $[12,13]$ can possess grammatical rules representing the correspondence of meanings and character strings that are considered as combinations of symbols. In a dynamical model for the evolution of prototypical category structure introduced in [8], agents can emit, receive and process sequences of words.

In order to understand the origin and the evolution of language, we should deal with the emerging process of such a symbol processing ability. To this end, 
we need a model of an agent that autonomously acquires the ability of symbolic cognitive activities for effectively studying the emergence and the evolution of linguistic communication with a constructive approach.

Mathematical and computational studies of symbol processing have been done in artificial intelligence and connectionism. The former has difficulty in the self-organisation and the emergence of symbols, since symbols and syntactic rules governing the symbol processing are usually given by hand. While the latter can acquire, in part, the symbolic representation from scratch without preparing explicit symbolic elements, it is not good at explicitly describing symbols and their processing rules, since symbols have a distributed representation in neural networks. Thus, a new approach for symbol formation has been required [7]. A new approach is to integrate both artificial intelligence type and connectionism type methods ${ }^{1}$.

A recent development is to view cognitive systems from the dynamic perspective. van Gelder [18] argued that cognitive systems can be well understood by considering them as dynamical systems and has presented many examples of dynamic cognitive models [19]. This viewpoint is also proposed to describe dynamic aspects of brain activities using the framework of dynamical systems and chaos [17].

Chaos is a deterministic unpredictable dynamics - in spite that the time evolution of a system is fully described by deterministic equations, no one can predict its long term behaviour. The unpredictability is caused by the expansion of small differences by the nonlinearity of chaotic systems. This property is described as "sensitive dependence on initial conditions". Note that chaotic dynamics is not random but has certain structure temporally and geometrically. A geometrical structure of chaotic dynamics is often characterised by a "strange attractor", which is a limit set of orbits (attractor) having a fractal structure. Namely, chaos can bring fertile spaciotemporal structures into existence. Recently such interesting features of chaos has been payed attention as carrying brain functions $[16,17]$, and actually chaotic dynamics are found in real neurons and brains $[2,6]$.

The purpose of the present study is to construct a model of an agent showing symbolic cognitive activities with a dynamical system. When we construct a model of a cognitive agent for linguistic communication, we take the dynamic viewpoint not only for the cognitive systems [18] but also for language $[8,9,18]$. The dynamic view for language means that symbols are not mere correspondences of words to referents and symbol formation is not merely an assigning process of words to some objects.

The rest of this paper is organised as follows. In $\S 2$ we discuss how symbolic systems are able to be interpreted in dynamical terms. Based on the discussion, we introduce a model of dynamical systems for symbolic activities in $\S 3$, based

\footnotetext{
${ }^{1}$ Note that using a connectionist model does not necessarily mean that no symbolic element is involved. For example, in the simple recurrent network introduced by Elman [5], sequences of words which are discrete representations are fed to the network as inputs.
} 
on the Hopfield model. Concretely, the model is composed of coupled chaotic dynamical systems, called NZ maps. The simulation results of the model are shown in $\S 4$. We discuss the results in $\S 5$ and conclude this paper in $\S 6$.

\section{Symbol Systems as Dynamical Systems}

To model the symbolic activities in the framework of dynamical systems, we consider features of symbols. In general, symbols are considered to represent or to signify something and are manipulated according to some rules such as a grammar in language or a deduction rule in calculation and formal thought.

Harnad [7] summarised the features of symbol systems with the following definitions:

1. A symbol is a set of arbitrary physical tokens that are

2. manipulated on the basis of explicit rules

3. that are likewise physical tokens and strings tokens.

4. The rule-governed symbol-token manipulation is based purely on the form ${ }^{2}$ of the symbol tokens, i.e. it is purely syntactic, and

5. consists of rulefully combining and recombining symbol tokens.

6. There are primitive atomic symbol tokens and

7. composite symbol-token strings.

8. The entire system and all its parts are all semantically interpretable: the syntax can be systematically assigned a meaning.

This definition describes an external system that can be interpreted as symbolic rather than internal symbolic activities. In order to construct an agent model showing symbolic activities, we construe this definition as internal cognitive processes. Further, to implement the agent model using a dynamical system, we interpret the processes through the concepts of dynamical systems.

The items 1, 6 and 8 imply that there are some entities that are accepted or interpreted as representing something such as objects, states of affairs, or abstract ideas by cognitive agents. To receive some physical tokens a cognitive process recalls some memorised concepts. In the terms of dynamical systems, some inputs to a dynamical system bring it to certain (dynamic) states. This representative function is thought of as being realised by a kind of memory that is usually modelled by attractors of the dynamical system.

The items 2, 3 and 4 state that the cognitive agent performs a process of successive recall of concepts (memories) and the successions are rule-governed. In dynamical terms, there are (spontaneous) transitions among attractors and a transition is rule-governed or, at least, ordered.

The items 5, 7 and 8 mean that a part of sets or some series of physical tokens, but not all sets and series, are accepted as an ordered combination of entities, not as independent entities, and receiving processes of such series induce retrieval

${ }^{2}$ In Harnad's original article [7], the term "shape" is used. We reword this as "form" for clarification. 
processes of concepts. These activities are considered as evocations of ordered transitions among attractors by some input sequences in dynamical systems.

A chaotic neural network is a candidate to implement the above mentioned behaviour as a dynamical system. In chaotic neural networks, memories are realised as attractors of the system [1]. In some chaotic dynamical systems, transitions among "attractor ruins" have been found [11], that is called "chaotic itinerancy". Thus, we may be able to construct a system with plastically learnable symbolic activities by a chaotic dynamical system by introducing the following correspondences:

- symbols to attractors,

- symbol manipulations to transitions among attractors,

- manipulation rules, or regularities in manipulation, to order of the transition.

In the followings, we try to construct a dynamical system having the above mentioned properties corresponding to symbolic activities, that is, multiple attractors, transitions among the attractors, and order in th transitions. We will enquire if the dynamical system introduced in the next section has actually such properties by computer simulations.

\section{Model of Chaotic Neural Newtork}

\subsection{Coupled NZ map}

Nozawa [15] derived a chaotic neural network model from the Hopfield model [10] by introducing small negative self-feedback connections and by discretising the time variable using Euler method. The chaotic neural network model is called coupled NZ maps ${ }^{4}$. It is shown that this system chaotically itinerates among attractor ruins. Similar to the Hopfield model [10] and other chaotic neural networks [1], the coupled NZ maps can be used as an associative memory device. Nozawa also demonstrated the high information processing ability of this system in solving a class of combinatorial optimisation problem [15] and a nonlinear optimisation [14].

In this paper, we use the coupled NZ maps for a model representing the features of symbols discussed in the previous section. The coupled NZ maps is

\footnotetext{
${ }^{3}$ An attractor ruin is a region in a state space of a dynamical system, in which an orbit stays for a while like an attractor, but does not stay forever, and escapes from there.

${ }^{4}$ Because of the discretisation of the time variable, the model consists of difference equations, that is, maps, while the original Hopfield model consists of differential equations. In a network model the elements described by a single NZ map interact through connections, therefore the elements are said to be "coupled" with each other in a neural network system.
} 
given by the following equations ${ }^{5}$ :

$$
\begin{aligned}
p_{i}(t+1) & =F_{q_{i}(t)}\left\{p_{i}(t)\right\}, \\
q_{i}(t) & =-\frac{1}{T_{i i}}\left\{\sum_{j \neq i}^{N} T_{i j} p_{j}(t)+I_{i}\right\}, \\
F_{q}(p) & =r p+(1-r)\left[1-\frac{1}{2}\left\{1+\tanh \left(\frac{p-q}{2 \beta}\right)\right\}\right],
\end{aligned}
$$

where the symbols in these equations are as follows:

- $p_{i}(t)$ : the internal buffer of the $i$ th neuron at time $t$, which develops according to the map, $F_{q_{i}(t)}$, defined by Eqn.(3)

- $q_{i}(t)$ : the influence from the other neurons to the $i$ th neuron as shown in Eqn.(2)

$-T_{i j}$ : the synaptic connection between the $i$ th and the $j$ th neurons

$-T_{i i}$ : the self-feedback connection of the $i$ th neuron

- $I_{i}$ : the threshold of the $i$ th neuron

$-N$ : the number of neurons

- $r$ : the parameter related to the damping constant of the neurons

$-\beta$ : the parameter related to the gain constant of the neurons ${ }^{6}$

In order to understand the basic properties of the $\mathrm{NZ}$ maps, let us look at the behaviour of single NZ map. When there is no connection between the elements, $T_{i j}=0$ for all $i$ and $j \neq i$. Therefore, $q_{i}(t)$ in Eqn.(2) is constant $q_{i}=I_{i} / T_{i i} \equiv q$. Thus the single NZ map is described by

$$
p(t+1)=r p+(1-r)\left[1-\frac{1}{2}\left\{1+\tanh \left(\frac{p-q}{2 \beta}\right)\right\}\right] .
$$

This map is a combination of a linear function $p$ and a reversed sigmoid function with the combination coefficient $r$. The parameter $r$ usually takes a value between 0 and 1, thus the map has three branches as shown in Fig. 1. When an orbit comes to the middle branch, the expansion of small differences occurs and the dynamics comes to be chaotic, since the slope of this branch is steep. Because the variable $q$, which is the ratio of the threshold to the self-feedback connection, moves the threshold of the sigmoid function, the middle branch of the single NZ map moves with the value of $q$. Namely, the variable $q$ plays a role of a control parameter to determine the shape and the nonlinearity of the single map. When $q$ is 0 and 1 , the map has a fixed point around $p=0$ and $p=1$, respectively. When $0<q<1$, the orbit shows chaotic behaviour. Examples of the map and the dynamics for different values of $q$ are shown in Fig. 1. In Fig. 1a) and c), the dynamics are chaotic for $q=0.09$ and 0.9 , respectively, and in Fig. 1b), period two dynamics is shown for $q=0.5$.

\footnotetext{
${ }^{5}$ Refer [15] for the detailed derivation from the Hopfield model.

${ }^{6}$ As describe later, the parameters $T_{i i}, I_{i}, r$ and $\beta$ are chosen for the system to show chaotic behaviour and $T_{i j}$ is determined to store some memory patters in the system.
} 
a) $q=0.09$

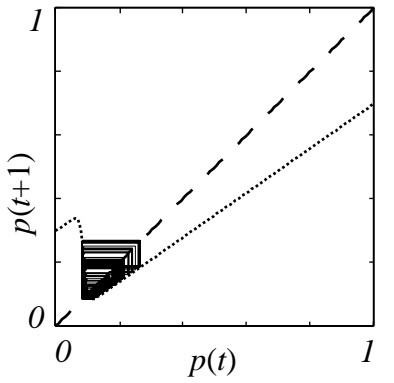

b) $q=0.5$

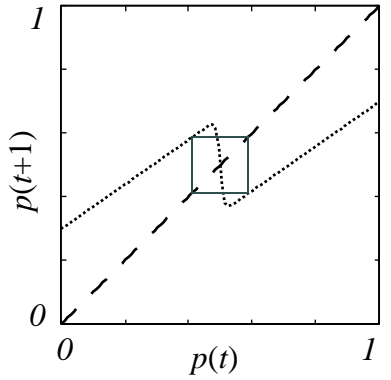

c) $q=0.9$

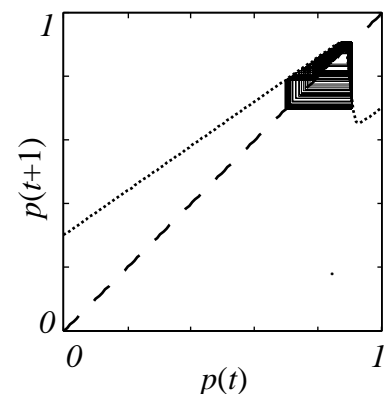

Fig. 1. The shapes of the single NZ map, Eqn.(4) and their dynamics for different values of the control parameter a) $q=0.09$, b) $q=0.5$ and c) $q=0.9$. The other parameters are $r=0.7$ and $\beta=0.006$. The horizontal and vertical axes are the value of the variable $p$ at time $t$ and $t+1$, respectively. The dotted lines are the shape of the map. The solid lines shows the dynamics (orbit) of the map (the cobweb plot). The dashed lines are the diagonals. If the orbit does not come to the middle branch which has steep negative slope, as in b), the dynamics is periodic. When the orbit comes to the middle branch, it shows chaotic motion as a) and c)

In the coupled system, the control parameter $q$ changes with time by the influences from other elements, as Eqn.(2) says. Even if the dynamics of some elements fall into fixed points, the values of $q$ of such elements are changed by the other ones through the connections, and then the orbits can escape from the fixed points. Therefore, the coupled NZ maps consists of a variety of maps of all sorts of dynamical motions, that induces diverse dynamic behaviour of the system, such as fixed, periodic and chaotic motions and transitions among such states.

\subsection{Embedding Patterns as Attractors}

As mentioned above, we consider that symbols correspond to attractors of a dynamical system. Since the system introduced here is basically an associative memory model, we can embed several memory states as attractors of the dynamical system [10]. To do this, the connection weights between elements should be appropriately set or learnt corresponding to patterns to be embedded, or memorised.

In order to embed a pattern represented by a $N$ dimensional vector $\boldsymbol{V}^{s}=$ $\left(V_{1}^{s}, \cdots, V_{N}^{s}\right)$ with $V_{i}^{s} 1$ ("ON") or 0 ("OFF"), where the index $s$ indicates each embedded pattern, the following equation is used to determine the connection $T_{i j}$ between the $i$ th and the $j$ th elements,

$$
T_{i j}=\sum_{s}\left(2 V_{i}^{s}-1\right)\left(2 V_{j}^{s}-1\right)
$$




\subsection{Recalled Pattern}

The coupled system behaving according to Eqns.(1) (3) represents some patterns. We define a recalled pattern $\phi(t)=\left\{\phi_{1}(t), \cdots, \phi_{N}(t)\right\}$ by observing the values of $q_{i}(t)$ of all elements as

$$
\phi_{i}(t)= \begin{cases}1 & \left(q_{i}(t) \geq \bar{q}(t)\right), \\ 0 & \left(q_{i}(t)<\bar{q}(t)\right),\end{cases}
$$

where

$$
\bar{q}(t)=\lim _{t \rightarrow \infty} \frac{1}{t N} \sum_{t^{\prime}=0}^{t-1} \sum_{i=1}^{N} q_{i}\left(t^{\prime}\right)
$$

is the criterion to separate whether each element is "ON" $\left(\phi_{i}(t)=1\right)$ or "OFF" $\left(\phi_{i}(t)=0\right)$. This criterion is the spaciotemporal average of $q_{i}(t)$ [15]. When a recalled pattern coincides with one of the embedded patterns, that is,

$$
\phi(t)=\boldsymbol{V}^{s}
$$

for some $s$, the embedded pattern, or memory, is retrieved.

\section{Simulation Results}

We embed three orthogonal patterns, shown in Fig. 2 and named $C, F, 4$, respectively, in the system with $N=16$ elements. Because of the symmetrical nature of the system, the reversed patterns of the embedded ones are also attractors. Such reversed patterns are labelled as $\bar{C}, \bar{F}$ and $\overline{4}$, respectively. All the patterns other than the embedded and their reversed patterns are treated in a lump and labelled as $O$. The parameters are $q=I_{i} / T_{i i}=0.09, r=0.7, \beta=0.006$ throughout the experiments described in this paper. This parameter set means that each element alone keeps showing chaotic behaviour as depicted in Fig. 1a). In the following experiments, we change the strength of the self-feedback connection, $T_{i i}$, of the all elements as a control parameter. Note that the ratio of $T_{i i}$ to $I_{i}$ is fixed.
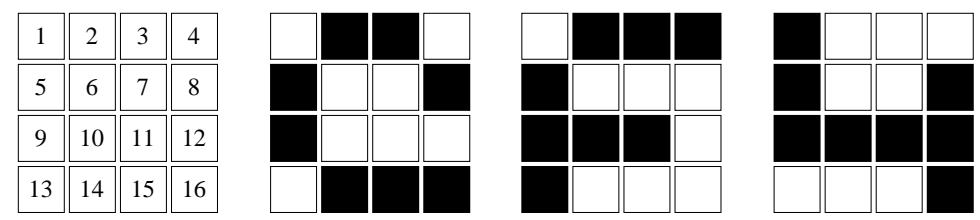

Fig. 2. Schematic view of embedded patterns. The black and white boxes mean 1 ("ON") and 0 ("OFF"), respectively. The patterns are named as $C, F$ and 4 , respectively. The index for each elements is arranged as the left most figure. 


\subsection{Recall and Transition of Embedded Patterns}

The system starts from a generic initial state. Then in a certain region the strength of the self-feedback connection, $T_{i i}$, causes the recall of one of the embedded or their reversed patterns after a certain time (Fig. 3). The two graphs in Fig. 3 have the same parameters but differ in their initial states. They converge to different patterns. The fact that different initial conditions end up with different converged attractors means that this system has multi-attractors.
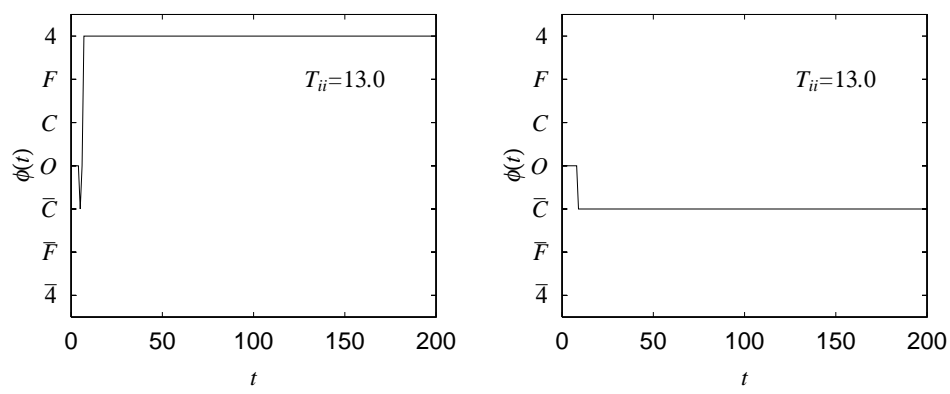

Fig. 3. The time series of recalled patterns. The $x$ and $y$ axes are time $t$ and the recalled patterns $\phi$, respectively. The labels $C, F, 4$ are the embedded patterns and $\bar{C}, \bar{F}, \overline{4}$ are their reversed patterns, respectively. The label $O$ means that the system is not in any embedded pattern. Two graphs starts from the different initial conditions. The self-feedback connection is $T_{i i}=13.0$ in the both graphs.

When we raise the strength of the self-feedback connection from the convergence parameter region, the system itinerates among embedded patterns through non-memorised patterns as shown in Fig. 4. Since there is no input to the system, the system autonomously changes its recalling patterns.

\subsection{Response to Input}

We examine how the system reacts to external inputs. Among a great variety of ways to input signals, we consider, as the simplest cases, constant and periodic inputs and observe the response of the system to these inputs. The external input $S(t)$ is given in Eqn.(2) as

$$
q_{i}(t)=-\frac{1}{T_{i i}}\left\{\sum_{j \neq i}^{N} T_{i j} p_{j}(t)+S(t)+I_{i}\right\} .
$$

In order to observe the dynamics of the system more precisely than just the sequence of recalled patterns, we introduce a distance measure of orbit $q_{i}(t)$ from the embedded patterns $\boldsymbol{V}^{s^{\prime}}$. The index $s^{\prime}$ is for the patterns both embedded and 


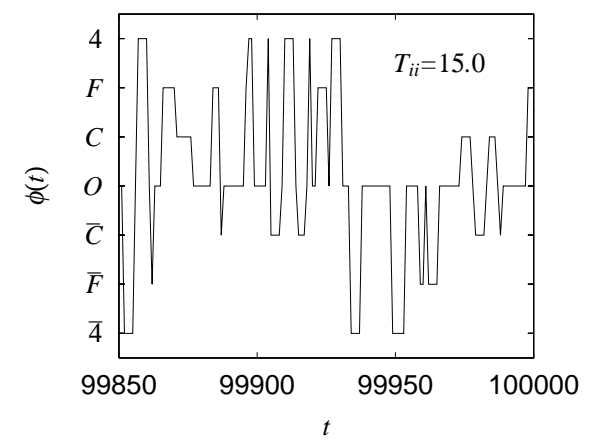

Fig. 4. The time series of recalled patterns $\phi$ without input. The $x$ axis is time. The self-feedback connection is $T_{i i}=15.0$. This graph shows an itinerant motion among the embedded patterns.

their reversed, that is, $C, F, 4$. and $\bar{C}, \bar{F}, \overline{4}$, while $s$ in Eqn.(5) indicates only the embedded patters. The measure is defined as

$$
\operatorname{Dist}^{s^{\prime}}(t)=\sqrt{\sum_{i=1}^{N}\left(V_{i}^{s^{\prime}}-q_{i}(t)\right)^{2}} .
$$

When any embedded and its reversed pattern is not definitely recalled, the orbit is categorised merely as $O$, as seen in Figs. 3 and 4 .

Constant Input We give a constant input sequence $S(t)=0.3$ at $t=10000 \sim$ 20000 for the system with the self feedback connections $T_{i i}=15.0$ for the all elements. While the system shows the itinerant motion when there is no input as in Fig 4, the constant input brings the system sometimes to become fixed to a pattern and sometimes to fluctuate among the patterns. Figure 5(Left) shows the time series of $q_{i}(t)$ around the input when the system falls onto a fixed pattern. The elements fluctuate widely, they itinerate among attractors before the input is given, and are then stabilised by the input.

Figure 5(Right) is a magnification of the dynamics of the system around the beginning of the input. Injecting the constant input causes the orbits showing chaotic dynamics to separate into two clusters. The dynamics are still chaotic but this is a transient state, and after 50 steps from the beginning of the input, the orbits of all elements enter stable periodic motions.

The elements are actually clustered hierarchically. Eight of 16 elements have higher values of $q_{i}(t)$ than the other ones as shown in Fig. 6(Left). The former elements, $i=1,6,7,8,12,14,15,16$ with numbering from top left to bottom right in Fig. 2, correspond to "ON" elements of the pattern $\bar{F}$. They form 4 sub-clusters consisting of two elements each. The residual 8 elements in the 

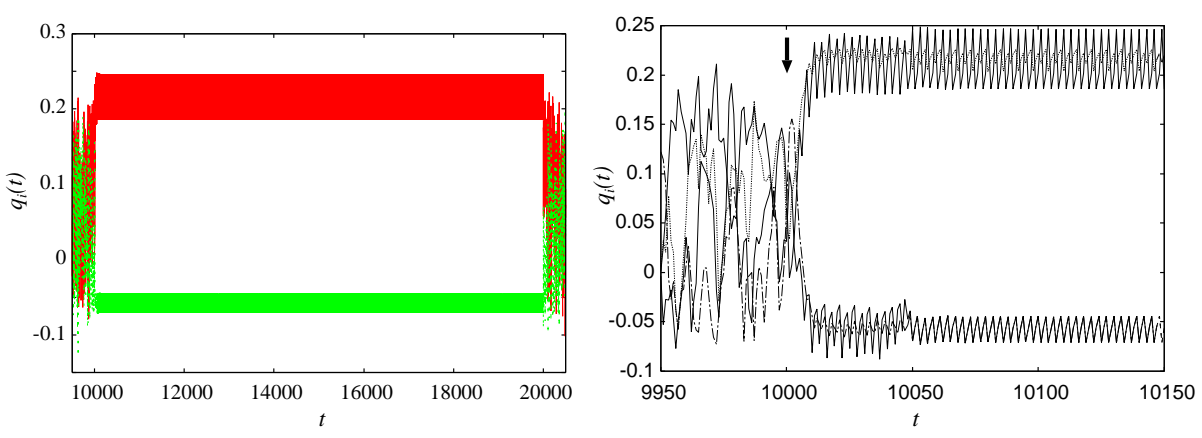

Fig. 5. (Left) The time series of $q_{i}(t)$ when a fixed input $S(t)=0.3$ in Eqn. (9) is given. The $x$ axis is time. The self-feedback connection is $T_{i i}=15.0$. The time series of 2 elements among 16 are drawn. (Right) The time series of $q_{i}(t)$ around the beginning of the input. The time series of 4 of 16 elements are depicted. The orbits, chaotically fluctuating before the input, promptly split into two clusters, when the input starts (indicated by the down arrow). Then, the dynamics are stabilised at a periodic state after 50 steps from the beginning of the input.

latter cluster, corresponding to "OFF" elements, synchronously oscillate while the input is given. Although the elements are not fixed by the dynamics of $q_{i}(t)$, the nearest pattern does not change. The time series of the distance measure, depicted in Fig. 6(Right), tells that the system stays at a state where the nearest pattern is $\bar{F}$.

As we mentioned, the system with a constant input sequence sometimes converges to various fixed patterns and sometimes itinerates among the embedded patterms and their reverses, in which case itinerant motion is not the same as one without an input sequence. This behavioural diversity depends on the timing of the input, since the system is in an itinerant motion as shown in Fig. 4. This itinerant behaviour is considered as the internal dynamics of the system. The system differs in its response to stimuli according to its internal dynamics, even though the same stimulus is given.

Sinusoidal Input We input a sinusoidal sequence,

$$
S(t)=A \sin (2 \pi \omega t),
$$

at $t=10000 \sim 20000(A=0.7, \omega=0.001)$ to the same system as in the previous experiment, the self-feedback connections are $T_{i i}=15.0$ for all the elements. A transition among the patterns is observed as shown in Fig. 7.

For a closer observation, we draw the dynamics of the distance measure in Fig. 8. This graph tells us that the change of the nearest patterns occurs with roughly the same intervals. The interval approximately matches with the cycle of the sinusoidal input. The order of recalled patterns is not periodic. Further, we have not found clear statistical order in the transition among recalled patterns. 

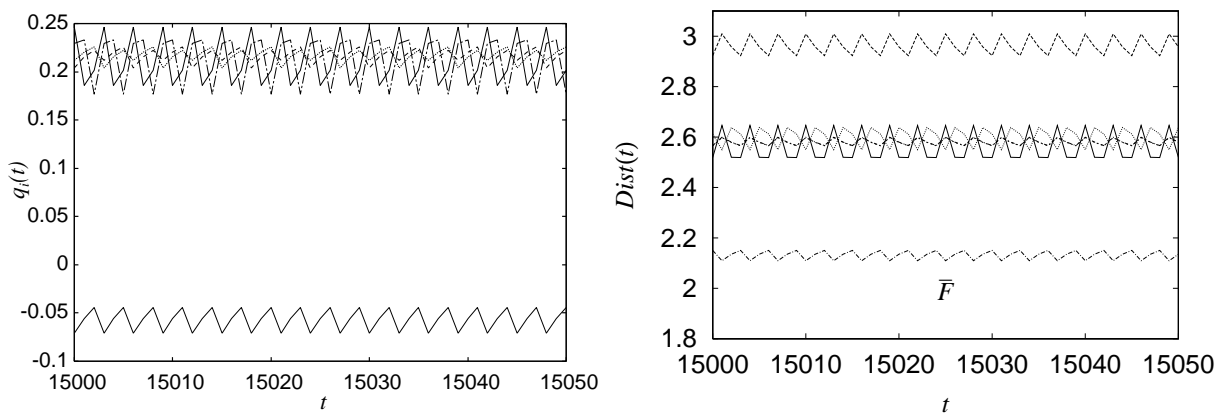

Fig. 6. (Left) The time series of $q_{i}(t)$ around the mid of the inputting period when a fixed input $S(t)=0.3$ is given. The $x$ axis is time. The self-feedback connection is $T_{i i}=15.0$. Note the change in scale of $t$ on the $x$ axis from Fig. 5(Right). The time series of all 16 elements are superimposed, but only 5 lines are distinguishable. The elements form two clusters. The upper cluster consisting of 8 elements corresponds to a pattern, $\bar{F}$. The elements are subdivided into 4 clusters, each of which has two elements. The residual 8 elements in the lower cluster have the same values while the input is given. (Right) The time series of the distance measure from the embedded patterns, Dist ${ }^{s^{\prime}}(t)$. The six orbits of the distance from all patterns are superimposed. The orbits periodically change, but the nearest pattern is fixed at $\bar{F}$.

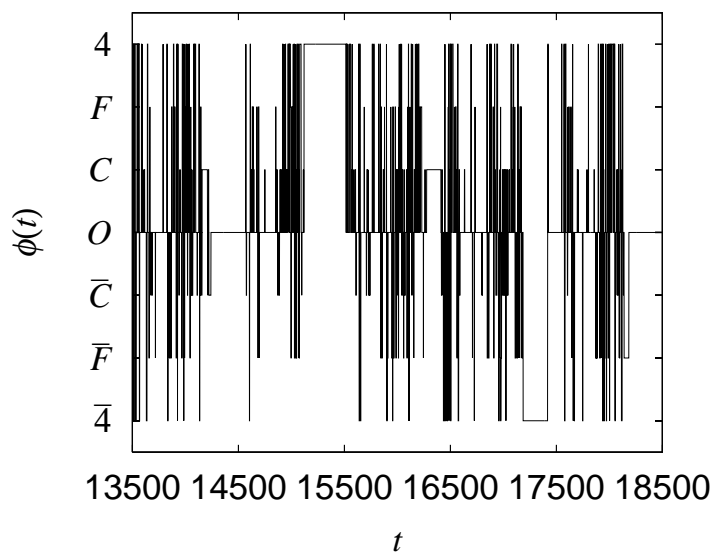

Fig. 7. The time series of recalled patterns with a sinusoidal input sequence. The $x$ axis is time. The self-feedback connection is $T_{i i}=15.0$. This graph shows an itinerant motion among the patterns. 


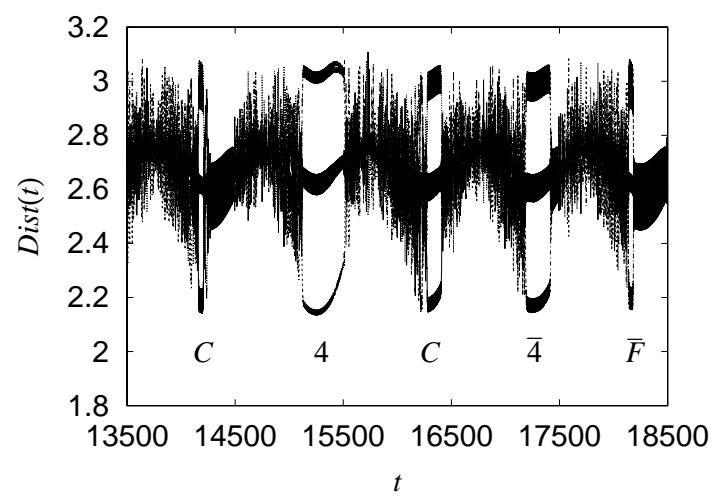

Fig. 8. The time series of the distance measure from embedded patterns, Dist ${ }^{s^{\prime}}(t)$, when a sinusoidal input sequence is given. The $x$ axis is time. The six orbits of the distance from all patterns are superimposed. The self-feedback connection is $T_{i i}=15.0$. A transition among the nearest patterns with nearly the same intervals is observed.

The change of the recalled pattern is certainly induced by the periodicity of the sine wave. This is clearly perceived from Fig. 9. When the amplitude of the sine wave falls below zero, the clustering (synchronisation or recalling a pattern) is dissolved and the system enters a chaotic (itinerate) state. When the amplitude of the input become positive, the system starts to synchronise again and is attracted to a pattern. But a memory of the last recalled pattern (the time correlation between recalled patterns) is destroyed by the interleaved chaotic motion. Thus, there is no ordered transition and a transition rule among the patterns is not formed.

Figure 9 shows the existence of intrinsic instability of the system, as well. The clustering is destroyed even though the amplitude of the input is large enough around $t=11200$. This is the effect of internal chaotic dynamics.

\section{Discussion}

We have suggested correspondences between symbolic activities and dynamical systems such that symbols correspond to attractors, symbol manipulations to transitions among attractors, and regularities in symbol manipulation to the order of transitions. Let us examine how the simulation results of the coupled $\mathrm{NZ}$ maps conducted in this paper are concordant with these correspondences, and therefore appropriate as a model of a symbolic cognitive agent.

At first, some patterns are embedded in the system and they are retrieved as attractors. The embedded patterns are recalled when a constant input is given to the system. Namely, the patterns and input sequences are associated like memories and some patterns of perceptions. This representation is an important function of symbols. Further, embedding several patterns or having multi-attractors 


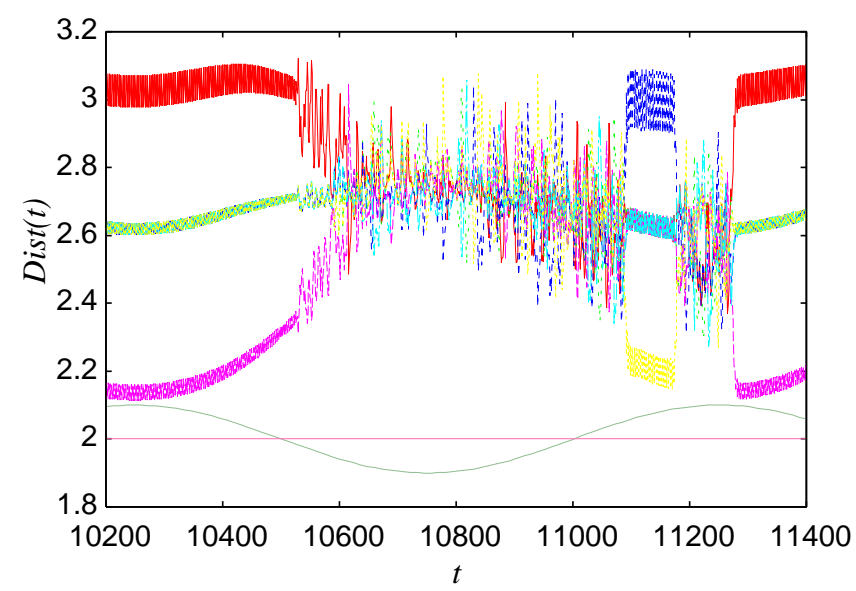

Fig. 9. The time series of the distance measure from the all embedded and their reversed patterns, Dist ${ }^{s^{\prime}}(t)$, with the sinusoidal input, $S(t)=A \sin (2 \pi \omega t)$ (lower periodic line). The amplitude of the input is transformed appropriately. The straight line shows the zero level of the input.

indicates that the system has the capacity to learn some number of symbols. The number of symbols is below the half of the system size $N$.

Concerning the second point, a dynamical system model of a symbolic cognitive agent is required to show, at least, transition among attractors. We can realise such behaviour in some parameter regions of the self-feedback connection $T_{i i}$ as shown in Fig. 4. The transition is evoked by the input sequence of an ordered change. Namely, our system also has a capacity for the symbol manipulations.

However, the transitions among attractors are not orderly. We have not found regularities in the transitions, that is, no basis for syntax. In chaotic dynamical systems, in general, time correlation decays exponentially. The rapid decrease of the time correlation causes these disordered transitions. The low correlation seems to be brought about partly by the orthogonal embedded patterns created by hand. If so, system learning in a particular environment with some structure may overcome this inadequacy. Namely, the order in the external world might form a structural coupling with the internal structure of the agent. Thus, to examine the system's status as a model of symbolic cognitive agent, it is important to investigate the behaviour of the system taught in a structured environment.

Let us further discuss the process of symbol formation or development of symbols based on the correspondences between symbolic activities and the behaviour of dynamical systems. Harnad [7] summarises the developmental process of representation as a progress from iconic representation to categorical and to symbolic. The iconic representation is demonstrated by retrieving an attractor from an input. This is shown by our system. 
The categorical representation can be translated in two ways. One is retrieving an attractor for different inputs, the other is grouping of attractors according to some features, such as dimension and nonlinearity. Our system shows the former behaviour. The dynamics of each element differ for different inputs but some dynamics are categorised into one pattern that is nearest to the dynamics. Namely, though precise internal states of the agent are not the same the representing symbol is the same for a class of inputs. To observe the latter interpretation, we need further investigation into the characteristics of attractors of the model.

The symbolic representation should show an orderly transition among attractors induced by an input sequence. As we found, this is not realised in the present system. If, however, we develop the system in a structured environment, the system may learn some symbols as attractors and show ordered transition among the attractors. Thus, we may be able to progress along the path of symbolic representation.

\section{Conclusion}

We have proposed a dynamical system model of a cognitive agent that can exhibit a part of symbolic behaviour using coupled chaotic maps, called NZ maps. We have shown that attractors of the dynamical system can represent symbols that can be embedded. The system can have internal dynamics and show symbol manipulation behaviour as transitions among the embedded attractors according to sequences of external input signals. However, the system did not show ordered transitions among symbols, that is, no basis for syntactically structured behaviour. Despite this drawback with the status quo, we conclude that the coupled NZ map system can be developed as a model of symbolic individuals, since we may overcome such insufficiency by further investigations, especially learning and developing in a particular structured environment.

\section{Acknowledgements}

This research is supported by JAIST research grant (grant for in-house research projects). We thank Caroline Lyon for her helpful comments and critical editing the manuscript.

\section{References}

1. Masaharu Adachi and Kazuyuki Aihara. Associative dynamics in a chaotic neural network. Neural Networks, 10:83-98, 1997.

2. Kazuyuki Aihara and Gen Matsumoto. Chaotic oscillations and bifurcations in squid giant axons. In A V. Holden, editor, Chaos, pages 257-269. Princeton University Press, 1986.

3. Angelo Cangelosi and Domenico Parisi, editors. Simulating the Evolution of Language. Springer, 2002. 
4. Terrence Deacon. The Symbolic Species: The Co-Evolution of Language and the Brain. W. W. Norton, 1997.

5. Jeffry L. Elman. Language as a dynamical system. In Robert Port and Timothy van Gelder, editors, Mind as Motion: Dynamical Perspectives on Behavior and Cognition, pages 195 - 225. MIT Press, 1995.

6. Walter J. Freeman. Simulation of chaotic EEG patterns with a dynamic model of the olfactory system. Biological Cybernetics, 56:139-150, 1987.

7. Stevan Harnad. The symbol grounding problem. Physica D, 42:335-346, 1990.

8. Takashi Hashimoto. The constructive approach to the dynamical view of language. In Angelo Cangelosi and Domenico Parisi, editors, Simulating the Evolution of Language, pages 307-324. Springer, 2002.

9. Takashi Hashimoto. Language as dynamics, - a computational study of ontogenetic and glossogenetic loop. In James R. Hurford and Tecumseh Fitch, editors, Fourth International Conference on the Evolution of Language - Proceedings, page 51, 2002.

10. John J. Hopfield. Neurons with graded response have collective computational properties like those of two-state neurons. Proc. Natl. Acad. Sci. USA, 81:30883092, 1984.

11. Kunihiko Kaneko and Ichiro Tsuda. Chaotic itinerancy. Chaos, 13(3):926-936, 2003.

12. Simon Kirby. Learning, bottlenecks and evolution of recursive syntax. In Ted Briscoe, editor, Linguistic Evolution through Language Acquisition, pages 173-203. Cambridge University Press, 2002.

13. Simon Kirby and James R. Hurford. The emergence of linguistic structure: an overview of the iterated learning model. In Angelo Cangelosi and Domenico Parisi, editors, Simulating the Evolution of Language, pages 121-147. Springer, 2002.

14. Noriyuki Matsuo and Hiroshi Nozawa. Coupled maps and nonlinear optimization (in japanese). In Proceedings of The Institute of Electrical Engineers of Japan (IEEJ), 1997.

15. Hiroshi Nozawa. A neural network model as a globally coupled map and applicatoins based on chaos. Chaos, 2(3):377-386, 1992.

16. Christine A. Skarda and W. J. Freeman. How brains make chaos in order to make sense of the world. Behavioral and Brain Sciences, 10:161-195, 1987.

17. Ichiro Tsuda. Toward an interpretation of dynamic neural activity in terms of chaotic dynamical systems. Behavioral and Brain Sciences, 24(5):793-847, 2001.

18. Timothy van Gelder. The dynamical hypothesis in cognitive science. Brain and Behavioural Sciences, 10:615-665, 1998.

19. Timothy van Gelder and Robert Prot, editors. Mind as Motion: Dynamical Perspectives on Behavior and Cognition. MIT Press, 1995. 